\title{
DISK INSTABILITIES IN THE BLACK HOLE BINARIES
}

\author{
J. K. CANNIZZO \\ Universities Space Research Association \\ NASA/GSFC/Code 666, Greenbelt, MD 20771, USA
}

\section{Introduction}

I will briefly review recent progress in our understanding of the global propagation of accretion disk instabilities operating in interacting binary systems in which the accreting star is a $\sim 10 \mathrm{M}_{\odot}$ black hole. A detailed discussion of this work can be found in Cannizzo, Chen \& Livio (1995).

The X-ray light curves of the brightest and best studied X-ray novae systems such as A0620-00, GS2000+25, GS1124-68, and GRO J0422+32 - are notable in two respects: the rise times are fast (a few days) and the decays have an exponential character, where the $e$-folding time constant is about 30 to $40 \mathrm{~d}$ (Mineshige, Yamasaki, \& Ishizaka 1993, hereafter MYI). It is noteworthy that outbursts in dwarf novae also decay exponentially, with the time constant being about a factor of 10 shorter. In this contribution I will discuss the constraints on the physics of accretion disks which the (observed) exponential decay enables us to make.

\section{Discussion}

Why does the X-ray flux fade as the X-ray nova progresses? X-ray novae are thought to be powered by accretion onto a massive compact object, most probably a black hole $(\mathrm{BH})$, and therefore the fading must be due to a diminution of the rate of mass flow onto the $\mathrm{BH}$. On the assumption that the mass flow rate is determined by the reservoir of material available in the accretion disk, one might imagine that the rate of mass flux would become smaller as the mass of the disk decreases. Furthermore, from the exponential nature of the decay, we know that the rate at which the accretion disk mass is becoming smaller must vary with the disk mass: $\dot{M}_{\text {disk }}=-M_{\text {disk }} / \tau(\mathrm{MYI})$. The solution to this differential equation has the desired form $M_{\text {disk }}=M_{0} e^{-t / \tau}$, or $L_{\text {disk }} \propto \dot{M}_{\text {disk }} \propto e^{-t / \tau}$.

A. Evans and J. H. Wood (eds.), Cataclysmic Variables and Related Objects, 135-138.

(C) 1996 Kluwer Academic Publishers. Printed in the Netherlands. 
How can the mass of the accretion disk be made to decrease? The most obvious way is simply to allow accretion onto the $\mathrm{BH}$ to proceed at a faster rate than material is being resupplied into the outer disk from the mass losing star. Unfortunately, it turns out to be quite difficult with this simple mechanism to obtain the law $\dot{M}_{\text {disk }} \propto M_{\text {disk }}$. Many investigators have examined the problem of the nature of the decay of the light curve produced by loss of disk matter due to accretion, and, for a variety of parameterizations of the viscous dissipation, one finds that $\dot{M}_{\text {disk }} \propto M_{\text {disk }}^{p}$ where $p \neq 1$ (Mantle \& Bath 1983; Lyubarskii \& Shakura 1987; Cannizzo, Lee \& Goodman 1990; MYI). Disk depletion through accretion inevitably leads to power law decays of the accretion luminosity rather than exponential decays.

What about removing matter from the outer edge of the disk rather than the inner edge? Could this provide an additional degree of freedom to allow theorists to concoct a plausible physical way to get $\dot{M}_{\text {disk }} \propto M_{\text {disk }}$ ? The primary failing with the idea of taking matter out from the inner edge was that the rate of mass loss depends on the local viscosity. By removing matter from the outer edge we are not necessarily limited by this restriction. For example, in the limit cycle model for dwarf nova outbursts, there exists a strong outflow of matter from the inner disk to the outer disk due to the action of a cooling transition front. One finds the outflow in time dependent modeling of the limit cycle, and the outflow is caused by the existence of an interface between the material in the high viscosity state (in the inner disk) and the material in the low viscosity state (in the outer disk).

We do not know a priori whether the action of the cooling front can lead to an exponential decay of the accretion luminosity. No one has attempted a numerical study in which some control parameter for the accretion disk physics is varied in a systematic way to see what conditions (if any) must exist so that $L_{\text {disk }} \propto e^{-t / \tau}$ during the decay. Our uncertainty about the physics of the viscosity in accretion disks - a topic which was covered so well by Chris Tout in his review talk - is customarily lumped into the ' $\alpha$-parameter' (Shakura \& Sunyaev 1973) wherein one sets the $\phi-r$ component of the stress tensor equal to $\alpha$ times the gas pressure. In order to obtain well separated outbursts for dwarf novae using the limit cycle model, most workers have found it necessary to increase $\alpha$ in going from the low to high viscosity state in the model. One of the popular functional forms for $\alpha$ has been $\alpha=\alpha_{0}(h / r)^{n}$, where $h$ is the local semi-thickness of the disk and $r$ is the local radius. In an attempt to test this law for $\alpha$, we ran numerical models in which different values for $n$ were tried. The results were quite interesting. For $n=1$ the decay in the accretion luminosity has a faster-than-exponential form. That is, when plotted as $\log L_{\text {disk }}$ versus time, the resulting light curve is concave down. For $n=2$ the decay has a slowerthan-exponential form - i.e., the light curve is concave up. For $n=1.5$ the 
decay is exponential, just as observed. Our first finding, therefore, is that, based on the observed exponential decay, the law $\alpha=\alpha_{0}(h / r)^{1.5}$ seems to be the preferred one. Further tests reveal that the $e$-folding time constant varies directly with the mass of the accreting object $M_{1}$, and inversely with the constant $\alpha_{0}$ which appears in the $\alpha$ scaling.

By following the evolution of surface density and disk temperature during the decay of an outburst, one sees that the physical process ultimately responsible for the nature of the decay is the speed of the cooling front. The law $\dot{M}_{\text {disk }} \propto M_{\text {disk }}$ occurs only for $n=1.5$ because, for that $n$ value, $\dot{r}_{\mathrm{F}} \propto r_{\mathrm{F}}$, where $r_{\mathrm{F}}$ is the radius of the front. In other words, an exponential decrease in the mass of the disk (or, more properly, in the mass of the hot portion of the disk), is caused by an exponential constriction of its outer radius $r_{\mathrm{F}}=r_{0} e^{-t / \tau^{\prime}}$.

By examining the width of the transition front, $w_{\mathrm{F}}$, in the time dependent computations, we find $w_{\mathrm{F}}=\sqrt{h_{\mathrm{F}} r_{\mathrm{F}}}$, where $h_{\mathrm{F}}$ and $r_{\mathrm{F}}$ are the disk semi-thickness and radius evaluated in the disk annulus lying just interior to the cooling front. This appears to be true regardless of how the viscosity is parameterized, and therefore seems to be quite general. The discovery of this law was made possible through the use of our high resolution code in which we divide the disk into 1000 radial elements. Most previous studies have taken $\sim 20 \ldots 40$ radial elements. Although we are confident that the relation $w_{\mathrm{F}}=\sqrt{h_{\mathrm{F}} r_{\mathrm{F}}}$ represents a fundamental property of the cooling front, we have yet to derive this relation from first principles.

The speed of the cooling transition front is given by

$$
v_{\mathrm{F}}=\dot{r}_{\mathrm{F}}=-\frac{\nu_{\mathrm{F}}}{w_{\mathrm{F}}}=-\frac{\alpha_{\mathrm{F}} h_{\mathrm{F}}^{2} \Omega_{\mathrm{F}}}{w_{\mathrm{F}}},
$$

where $\nu$ is the viscosity coefficient and again, the subscript $\mathrm{F}$ denotes values in the high viscosity disk just interior to the cooling front. If we adopt $\alpha=\alpha_{0}(h / r)^{n}$ and use $h=c_{\mathrm{s}} / \Omega$ (from hydrostatic equilibrium), we get

$$
\dot{r}_{\mathrm{F}}=-\alpha_{0} c_{\mathrm{s}}^{(n+3 / 2)}\left(\frac{r_{\mathrm{F}}}{G M_{1}}\right)^{(2 n+1) / 4} .
$$

For $n=1.5$ this reduces to

$$
\dot{r}_{\mathrm{F}}=-\frac{\alpha_{0} c_{\mathrm{s}}^{3}}{G M_{1}} r_{\mathrm{F}}
$$

or

$$
r_{\mathrm{F}}=r_{0} e^{-t / \tau^{\prime}},
$$

where $\tau^{\prime}=\left(G M_{1} / \alpha_{0}\right) c_{\mathrm{s}}^{-3}$. Note that the sound speed $c_{\mathrm{s}}$ was taken to be constant. This is because $T_{\mathrm{F}} \simeq 30000 \mathrm{~K}$ just interior to the cooling front, independent of radius, giving $c_{\mathrm{s}} \simeq 16 \mathrm{~km} \mathrm{~s}^{-1}$. 
The law $v_{\mathrm{F}}=\sqrt{h_{\mathrm{F}} r_{\mathrm{F}}}$ has turned out to be crucial for understanding why $n=1.5$ is special. Equation (2) shows that $\dot{r}_{\mathrm{F}} \propto r_{\mathrm{F}}$ only for $n=1.5$. Furthermore, for $n=1.5$ we also see that $\tau^{\prime} \propto M_{1} / \alpha_{0}$, which explains the numerical results regarding $M_{1}$ and $\alpha_{0}$ which were quoted earlier. By taking $M_{1}=10 \mathrm{M}_{\odot}$, we find $\alpha_{0} \simeq 50$ is needed to get $\tau \simeq 30 \mathrm{~d}$ as is observed. Meyer \& Meyer-Hofmeister (1984) used the exact same law we do, $\alpha=50(h / r)^{1.5}$, in modeling dwarf novae. These systems also possess outbursts with exponential decays, but with $\tau \sim 3 \mathrm{~d}$. This is expected if $\alpha_{0}$ were the same in both cases, because the primary masses are $\sim 1 \mathrm{M}_{\odot}$ in cataclysmic variables - of order a factor 10 smaller than those estimated for the X-ray novae (Shahbaz, Naylor \& Charles 1994; Shahbaz et al. 1994). Acknowledgement. This research was supported through the visiting scientist program under the Universities Space Research Association (USRA contract NAS5-32484) in the Laboratory for High Energy Astrophysics at Goddard Space Flight Center.

\section{References}

Cannizzo, J. K., Lee, H. M., Goodman, J., 1990, Ap. J., 351, 38

Cannizzo, J. K., Chen, W., Livio, M., 1995, Ap. J., 454, 880

Lyubarskii, Yu. É., Shakura, N. I., 1987, Soviet Astronomy Letters, 13, 386

Mantle, V. J., Bath, G. T., 1983, MNRAS, 202, 151

Meyer, F., Meyer-Hofmeister, E., 1984, A\&A, 132, 143

Mineshige, S., Yamasaki, T., Ishizaka, C., 1993, PASJ, 45, 707

Shahbaz, T., Naylor, T., Charles, P. A., 1994, MNRAS, 268, 756

Shahbaz, T., Ringwald, F. A., Bunn, J. C., et al., 1994, MNRAS, 271, L10

Shakura, N. I., Sunyaev, R. A., 1973, A\&A, 24, 337 